\title{
Boundaries and the Casimir effect in non-commutative space-time
}

\author{
R. Casadio, ${ }^{1,2, *}$ A. Gruppuso, ${ }^{3,2, \dagger}$ B. Harms,${ }^{4, \ddagger}$ and O. Micu ${ }^{4, \S}$ \\ ${ }^{1}$ Dipartimento di Fisica, Università di Bologna, via Irnerio 46, 40126 Bologna, Italy \\ ${ }^{2}$ I.N.F.N., Sezione di Bologna, via Irnerio 46, 40126 Bologna, Italy \\ ${ }^{3}$ I.N.A.F.-I.A.S.F. Bologna, via Gobetti 101, 40129 Bologna, Italy \\ ${ }^{4}$ Department of Physics and Astronomy, The University of Alabama, Box 870324, Tuscaloosa, AL 35487-0324, USA
}

\begin{abstract}
We calculate modifications to the scalar Casimir force between two parallel plates due to spacetime non-commutativity. We devise a heuristic approach to overcome the difficulties of describing boundaries in non-commutative theories and predict that boundary corrections are of the same order as non-commutative volume corrections. Further, both corrections have the form of more conventional finite surface effects.
\end{abstract}

PACS numbers: 02.40.Gh,11.10.Nx

\section{INTRODUCTION}

The idea that space-time coordinates do not commute below a certain length scale is a rather old one [1]. As such, it has been recently revived in string theory $[2,3]$, where one deals with spatially extended objects, and in the search for a quantum theory of gravity [4] where the Planck length naturally appears. Non-commutativity itself is a common feature of quantum theories. It is manifested in quantum mechanics in the phase-space commutation relations

$$
\left[p_{i}, x_{j}\right]=i \hbar \delta_{i j},
$$

and in quantum field theory in the commutation relations of creation and annihilation operators. Also Yang-Mills theories on non-commutative spaces [5] appear in string theory and M-theory.

Non-commutative geometry is based on the concept that there might exist a fundamental length (which we shall always denote as $\ell$ ) in the fabric of space-time [1]. For a parameter to be considered a fundamental length, it should respect Lorentz invariance, and therefore the time coordinate needs to be included among the noncommutative variables. However, this is not a trivial change and theories in which the time coordinate does not commute with spatial ones seem to be acausal. An example of such a theory is given in Ref. [3], where the authors study

\footnotetext{
*Electronic address: casadio@bo.infn.it

${ }^{\dagger}$ Electronic address: gruppuso@iasfbo.inaf.it

‡Electronic address: bharms@bama.ua.edu

$\S$ Electronic address: micu001@bama.ua.edu
}

the effects of space-time non-commutativity on the scattering of wave packets and show that stringy effects cancel the acausal effects that appear in field theories. Other motivations for non-commutativity come from D-brane scenarios $[2,3]$ where space non-commutativity appears when D-branes occur in hyper-magnetic fields, and time non-commutativity is generated similarly by nonzero hyper-electric fields [19].

Non-commutativity in space-time is usually implemented by replacing the ordinary product with the Moyal «-product [6]. One then finds that the free propagator is not changed, and a (self-)interaction in the field theory is necessary in order to see any corrections with respect to the commutative case (for a recent review, see e.g., Ref. [7]). An alternative, coherent state approach was proposed in Ref. [8] which leads to non-trivial corrections already at the level of free propagators, as one would more naturally expect (for some of its consequences, see Refs. [9, 10]).

The Casimir force between perfectly conducting (parallel) neutral plates per unit area [13] (we shall use natural units with $c=\hbar=1$ ),

$$
\mathcal{F}_{0}=\frac{F_{0}}{\mathcal{A}}=-\frac{\pi^{2}}{240 L^{4}},
$$

where $\mathcal{A}$ is the area of the plates and $L$ the separation between the plates $\left(\mathcal{A} \gg L^{2}\right)$, is perhaps the most prominent example of non-trivial vacuum effects in quantum electrodynamics. It has been studied extensively and can be measured with very high accuracy (for a review, see Refs. [14, 15]). Several papers have appeared in the literature which consider the corrections due to the existence of a minimal length [16] or of 
compactified extra spatial dimensions [17]. Because of the high accuracy of the measurements, the Casimir effect is also a natural candidate to show possible effects due to short-distance spacetime non-commutativity [18]. It is important to note that the final result in general depends on how non-commutativity is realized. For example, in the usual Moyal approach [6], noncommutative corrections will depend on the details of the (self)interaction terms which are included in the field theory [18]. In the approach of Ref. [8], which we shall follow, such corrections should not depend on coupling constants and therefore look universal [20].

In describing the Casimir force, one usually represents the plates as Dirac $\delta$-function sources or, equivalently, as boundary conditions on the field modes. Thus, a crucial point which needs to be addressed before introducing non-commutativity in the Casimir effect is how boundaries are described in a non-commutative space-time. ¿From a physical point of view, it is meaningless to assign a position with accuracy better than the minimum length admitted in the theory. Therefore what should be done is to give up the boundary conditions and, for example, describe the plates as sources "smeared" over a size of the order of the fundamental length $\ell$, making sure at the same time that the necessary conditions for having a discrete spectrum of modes between the plates continue to hold. Unfortunately, such prescriptions do not uniquely fix the interaction term in the Lagrangian which describes the plates explicitly, and it is hopeless to obtain exact expressions for the noncommutative modes given non-trivial potentials for the plates.

Based on the above observation, we shall therefore attempt a more modest approach by treating the non-commutativity around the boundaries "perturbatively" with respect to the standard treatment. This will allow us to show to what extent approximate calculations which make use of the usual boundary conditions can still be employed and find that, to leading order in the ratio between the non-commutative length and the plate separation, boundary corrections are expected to be of the same order as volume corrections and both corrections have the form of finite surface effects.

In Section II, we shall review the coherent state approach to quantum field theory in noncommutative space-time and then compute noncommutative corrections to the Casimir effect in Section III. We shall begin by discussing the problem of defining boundaries in a noncommutative space-time in Section III A where we estimate boundary effects and proceed with volume corrections in Section IIIB. The comparison with surface effects in the standard description will finally be discussed in Section IV and more conclusions drawn in Section V.

\section{NON-COMMUTATIVE SPACE-TIMES}

A $D$-dimensional non-commutative space-time can be defined in terms of space-time coordinates $x^{\mu}$ (where $\mu=1,2, \ldots, D$ ) which satisfy the following commutation relations:

$$
\left[x^{\mu}, x^{\nu}\right]=i \Theta^{\mu \nu},
$$

where $\Theta^{\mu \nu}$ must be an antisymmetric Lorentz tensor [8]. As such, it can be transformed into a block-diagonal form:

$$
\hat{\Theta}^{\mu \nu}=\operatorname{diag}\left(\hat{\Theta}_{1}, \hat{\Theta}_{2}, \ldots, \hat{\Theta}_{D / 2}\right),
$$

where

$$
\hat{\Theta}_{i}=\Theta_{i}\left(\begin{array}{cc}
0 & 1 \\
-1 & 0
\end{array}\right) .
$$

Since a coordinate reversal changes the sign of one of these $\Theta_{i}$, we can without loss of generality require that all $\Theta_{i}$ be positive.

In order to have full non-commutativity, one needs to work in a space-time that has an even number of dimensions. Then the $D=2 d$ coordinates can be represented by $d$ two-vectors:

$$
\begin{aligned}
\hat{x}^{\mu} & =\left(\hat{x}^{1}, \hat{x}^{2}, \ldots, \hat{x}^{2 d-1}, \hat{x}^{2 d}\right) \\
& =\left(\overrightarrow{\hat{x}}_{1}, \overrightarrow{\hat{x}}_{2}, \ldots, \overrightarrow{\hat{x}}_{d}\right),
\end{aligned}
$$

with $\overrightarrow{\hat{x}}_{i} \equiv\left(\hat{y}_{1 i}, \hat{y}_{2 i}\right)$ being two-vectors in the $i$-th non-commutative plane that satisfy

$$
\left[\hat{y}_{1 i}, \hat{y}_{2 i}\right]=i \Theta_{i} \text {. }
$$


In a coherent state approach a set of commuting ladder operators is constructed from noncommutative space-time coordinates only [8]. We define the ladder operators for the $i$-th plane in the following way,

$$
\begin{aligned}
& \hat{a}_{i}=\frac{1}{\sqrt{2}}\left(\hat{y}_{1 i}+i \hat{y}_{2 i}\right) \\
& \hat{a}_{i}^{\dagger}=\frac{1}{\sqrt{2}}\left(\hat{y}_{1 i}-i \hat{y}_{2 i}\right) .
\end{aligned}
$$

These operators will then satisfy the canonical commutation rules

$$
\left[\hat{a}_{i}, \hat{a}_{j}^{\dagger}\right]=\delta_{i j} \Theta_{i}
$$

Normalized $(\langle\alpha \mid \alpha\rangle=1)$ coherent states can now be defined for these operators as

$$
|\alpha\rangle=\prod_{i} \exp \left[\frac{1}{\Theta_{i}}\left(\bar{\alpha}_{i} \hat{a}_{i}-\alpha_{i} \hat{a}_{i}^{\dagger}\right)\right]|0\rangle,
$$

where $|0\rangle$ is the vacuum annihilated by all $\hat{a}_{i}$.

The usual commutative coordinates are associated with the non-commuting ones as their mean values over coherent states. In this way, a noncommutative plane wave can be calculated using Hausdorff decomposition, resulting in the following form

$$
\begin{aligned}
& \left\langle\alpha\left|\exp \left[i \sum_{i=1}^{d}(\vec{p} \cdot \overrightarrow{\hat{x}})_{i}\right]\right| \alpha\right\rangle \\
& =\exp \left\{-\sum_{i=1}^{d}\left[\frac{\Theta_{i}}{4}\left(p_{1 i}^{2}+p_{2 i}^{2}\right)+i(\vec{p} \cdot \vec{x})_{i}\right]\right\},
\end{aligned}
$$

where $p_{1 i}$ and $p_{2 i}$ are the momenta canonically conjugate to the space-time coordinates. Eq. (11) then shows that a plane wave in the non-commutative case will have damping factors proportional to $\Theta_{i}$ and that we recover the usual form in the limit $\Theta_{i} \rightarrow 0$. In a four-dimensional non-commutative space-time we have two such non-commutative planes $(d=2)$ and it is worth noting that, for complete space-time noncommutativity and $\Theta_{i}>0$, the damping factors are positive both for time and space momentum components, regardless if a Minkowski or Euclidean metric is used.

In the following, for simplicity, we will assume that the parameters which describe noncommutativity in the $i$-th plane in Eq. (7) are all the same, that is $\Theta_{i}=\Theta>0$, and denote with $\ell=\sqrt{\Theta}$ the natural length associated with non-commutativity.

\section{NON-COMMUTATIVE CASIMIR EFFECT}

We shall now calculate modifications to the scalar Casimir force due to the space-time noncommutativity described in the previous Section. Following Ref. [13], we shall do so by computing the vacuum energy density of a massless scalar field for the case of two perfectly conducting parallel plates separated by a distance $L$. Although in Casimir's original paper, the calculated Casimir force is due to the vacuum energy density of the electromagnetic field, several calculations have been carried out using scalar fields satisfying either Dirichlet or Neumann boundary conditions. For an extensive review on the subject, see Ref. [15].

The first issue we need to address is how to define the plates in a non-commutative theory, which will lead us to estimate "boundary" corrections due to the "fuzziness" of the plates. Subsequently, we shall also determine volume corrections which are present in the limit of sharply defined plates.

\section{A. Boundary corrections}

Let us begin by briefly reviewing how boundaries are taken into account in the standard commutative case. For this purpose, we consider the simple case of a (complex) massless scalar field $\phi=\phi(t, x)$ in two-dimensional Minkowski spacetime which interacts with two $\delta$-function potentials, at $x=0$ and $x=L$. The dynamics of this system is described by the usual Lagrangian density

$$
\mathcal{L}=\frac{1}{2}\left(\left|\partial_{t} \phi\right|^{2}-\left|\partial_{x} \phi\right|^{2}\right)+\mathcal{L}_{\text {int }},
$$

with

$$
\mathcal{L}_{\mathrm{int}}=-\frac{\lambda}{2 L} \delta(x)|\phi|^{2}-\frac{\lambda}{2 L} \delta(x-L)|\phi|^{2},
$$

where the coupling constant $\lambda$ is dimensionless [15]. Integrating around $x=0$ or $x=L$ 
one imposes the usual boundary conditions in which the jump of the first derivative of the field is related to the value of the field itself at the same point,

$$
\left.\Delta\left(\partial_{x} \phi\right)\right|_{y}=\frac{\lambda}{L} \phi(t, y)
$$

where $y=0$ or $L$,

$$
\left.\Delta\left(\partial_{x} \phi\right)\right|_{y}=\left.\partial_{x} \phi\right|_{y+\epsilon}-\left.\partial_{x} \phi\right|_{y-\epsilon},
$$

and the limit $\epsilon \rightarrow 0$ is understood. If we now take the coupling to infinity, Eq. (14), together with regularity of $\phi$ around $x=y$, enforces Dirichlet boundary conditions at the two points,

$$
\lim _{\lambda \rightarrow \infty} \phi(t, y)=0 \text {. }
$$

Upon introducing the usual Klein-Gordon scalar product

$$
\left(\phi_{n}, \phi_{m}\right)=\frac{i}{2} \int \mathrm{d} x\left(\phi_{n}^{*} \partial_{t} \phi_{m}-\phi_{m} \partial_{t} \phi_{n}^{*}\right),
$$

one then finds the normal modes

$$
\phi_{n}(t, x)=\sqrt{\frac{2}{n \pi}} e^{-i E_{n} t} \sin \left(\frac{n \pi x}{L}\right),
$$

with energy eigenvalues

$$
E_{n}=\frac{n \pi}{L},
$$

where $n$ is a positive integer.

When non-commutativity is taken into account, the $\delta$-functions describing the positions of the plates, should be replaced by smooth functions which differ from 0 in an interval of the order of the minimal length $\ell=\sqrt{\Theta}$ induced by non-commutativity. At the same time, we require these potential functions to preserve a discrete spectrum in between the plates. To achieve this, for instance, we can superimpose on each $\delta$ function a "box" potential,

$$
g(x)= \begin{cases}\ell^{-1} & \text { for }|x|<\ell \\ 0 & \text { for }|x|>\ell .\end{cases}
$$

The interaction term (13) will then be supplemented by

$$
\mathcal{L}_{\text {int }}^{\prime}=-\frac{\lambda^{\prime}}{2 L} g(x)|\phi|^{2}-\frac{\lambda^{\prime}}{2 L} g(x-L)|\phi|^{2},
$$

where $\lambda^{\prime}$ is also dimensionless [21]. Eq. (14) will correspondingly be replaced by

$$
\left.\Delta\left(\partial_{x} \phi\right)\right|_{y}=\frac{\lambda}{L} \phi(t, y)+\frac{\lambda^{\prime}}{L \ell} \int_{y-\ell}^{y+\ell} \mathrm{d} x \phi(t, x)
$$

In the strong coupling regime $\lambda \rightarrow \infty$, regularity of $\phi$ then requires that $\phi(t, 0)=\phi(t, L)=0$ as for the commutative case [see Eq. (16)]. The jump $\Delta\left(\partial_{x} \phi\right)$ is then proportional to $\lambda^{\prime}$, unless $\phi(t, x-y)=-\phi(t, y-x)$ around $y=0$ and $L$. This latter condition must strictly hold if one also wants to take $\lambda^{\prime} \rightarrow \infty$ in the strong coupling limit [otherwise $\Delta\left(\partial_{x} \phi\right)$ would diverge].

For finite values of $\lambda^{\prime}$, we can still use the potential (20) to estimate the non-commutative corrections by employing standard perturbation methods. In particular, we treat the interaction Lagrangian (21) as a perturbation with respect to (13) and estimate the correction to the energy levels of the strongly coupled $(\lambda \rightarrow \infty)$ unperturbed theory as

$$
\Delta E_{n}=\frac{\lambda^{\prime}}{L \ell} \int_{0}^{\ell} \mathrm{d} x\left|\phi_{n}\right|^{2} \sim \lambda^{\prime} E_{n}\left(\frac{\ell}{L}\right)^{2},
$$

where $\phi_{n}$ is given in Eq. (18) and the last approximation holds for $\ell \ll L$. A similar correction can be computed for the spectrum of continuous modes outside the plates. For example, to the left of $x=0$, such modes can be written as

$$
\phi_{k}(t, x)=\sqrt{\frac{2}{k W}} e^{-i k t} \sin (k x),
$$

where $W$ is a finite interval which must be taken to infinity at the end of the computation. The corresponding correction to the energy $E_{k}=|k|$ is then

$$
\Delta E_{k} \sim \lambda^{\prime} E_{k} \frac{\ell^{2}}{L W}
$$

which vanishes in the limit $W \rightarrow \infty$ and is thus negligible. This argument shows that noncommutative boundary corrections are negligible for continuous spectra, such as is the case of the moving mirror analysed in Ref. [10].

¿From the above results it follows that the 
Casimir force between "fuzzy" plates is given by

$$
\begin{aligned}
\mathcal{F}_{\text {f.p. }} & \simeq-\frac{\partial}{\partial L}\left[\sum_{n}\left(E_{n}+\Delta E_{n}\right)-W \int \mathrm{d} k E_{k}\right] \\
& \sim \mathcal{F}_{0}\left[1+3 \lambda^{\prime}\left(\frac{\ell}{L}\right)^{2}\right]
\end{aligned}
$$

that is

$$
\frac{\mathcal{F}_{\text {f.p. }}-\mathcal{F}_{0}}{\mathcal{F}_{0}} \sim\left(\frac{\ell}{L}\right)^{2}
$$

Of course, numerical factors were dropped from the final expressions since they would just be related to the specific form of the "fuzzy" potential (20). Further, had we considered more spatial dimensions, the difference would have appeared in an overall (volume) factor which could be absorbed in the couplings $\lambda$ and $\lambda^{\prime}$.

A few comments are now in order. Firstly, the correction in Eq. (26) explicitly depends on the coupling constant $\lambda^{\prime}$ and we have no means of estimating its value for the (unphysical) case of a scalar field considered here. One natural assumption would be to set $\lambda^{\prime} \sim 1$ and, for the more realistic case of an electromagnetic field, take $\lambda^{\prime} \sim \alpha$ (the fine structure constant). Further, since it is natural to assume that $\ell \ll L$ (otherwise non-commutativity would have already been discovered in our macroscopic world), our results will always be given in the perturbative expansion in powers of $\ell / L$ as we did above.

\section{B. Volume corrections}

Having estimated the effects due to the uncertainty in the position of the boundaries, we shall now compute volume corrections, that is the effects due to non-commutativity that remain if we impose the usual boundary conditions (16) corresponding to sharply defined plates.

Using the results reviewed in Section II, we can express a scalar field in a non-commutative four-dimensional Minkowski space-time in terms of the field modes as

$$
u_{\mathrm{NC}}(t, \vec{x})=\frac{e^{-\frac{\Theta}{4}\left(\omega^{2}+p^{2}\right)} e^{i \vec{p} \cdot \vec{x}-i \omega t}}{(2 \pi)^{3 / 2} \sqrt{2 \omega}},
$$

where $\vec{x}=(x, y, z), \omega=-p_{0}, \vec{p}=\left(p_{x}, p_{y}, p_{z}\right)$ and $p=\sqrt{\vec{p} \cdot \vec{p}}$. The fact that the time is noncommutative is reflected in the equation above by the presence of the energy $\omega$ in the Gaussian damping factor, and we recall that the relative sign between terms proportional to $\omega^{2}$ and $p^{2}$ in the exponential is not affected by the space-time signature.

The scalar field operator in terms of these field modes takes the form

$$
\hat{\phi}(t, \vec{x})=\int \frac{\mathrm{d}^{3} p}{(2 \pi)^{3 / 2} \sqrt{2 \omega}}\left[\hat{b}_{\vec{p}} e^{-\frac{\Theta}{4}\left(\omega^{2}+p^{2}\right)} e^{i \vec{p} \cdot \vec{x}-i \omega t}+\hat{b}_{\vec{p}}^{\dagger} e^{-\frac{\Theta}{4}\left(\omega^{2}+p^{2}\right)} e^{-i \vec{p} \cdot \vec{x}+i \omega t}\right],
$$

and the momentum density becomes

$$
\hat{\pi}(t, \vec{x})=\int \frac{\mathrm{d}^{3} p(-i \omega)}{(2 \pi)^{3 / 2} \sqrt{2 \omega}}\left[\hat{b}_{\vec{p}} e^{-\frac{\Theta}{4}\left(\omega^{2}+p^{2}\right)} e^{i \vec{p} \cdot \vec{x}-i \omega t}-\hat{b}_{\vec{p}}^{\dagger} e^{-\frac{\Theta}{4}\left(\omega^{2}+p^{2}\right)} e^{-i \vec{p} \cdot \vec{x}+i \omega t}\right]
$$

with the ladder operators satisfying

The massless free-field Hamiltonian

$$
\left[\hat{b}_{\vec{p}}, \hat{b}_{\vec{p}^{\prime}}^{\dagger}\right]=\delta^{(3)}\left(\vec{p}-\vec{p}^{\prime}\right)
$$

$$
H=\frac{1}{2} \int \mathrm{d}^{3} x\left[\pi^{2}+(\vec{\nabla} \phi)^{2}\right]
$$


then becomes

$$
\hat{H}=\int \frac{\mathrm{d}^{3} p}{(2 \pi)^{3}} \omega(p) e^{-\Theta \omega^{2}}\left(\hat{b}_{\vec{p}}^{\dagger} \hat{b}_{\vec{p}}+\frac{1}{2}\right),
$$

and the zero point energy

$$
\begin{aligned}
E_{\mathrm{ZP}} & \equiv\langle 0|H| 0\rangle \\
& =\int_{0}^{\infty} \frac{p^{2} \mathrm{~d} p}{(2 \pi)^{2}} e^{-\Theta p^{2}} \omega(p),
\end{aligned}
$$

which is finite since the Gaussian damping factor dominates at large $p$ and makes the above integral converge.

Let us assume that the two perfectly conducting plates are located at $x=0$ and $L$ and extend infinitely in the $(y, z)$-plane. The boundary conditions (16) then imply that, between the plates, the momentum along $x$ is quantized,

$$
p_{x}=\frac{\pi n}{L}, \quad n \in Z,
$$

whereas $\vec{k}=\left(p_{y}, p_{z}\right)$ takes continuous values. The frequency of a massless scalar field mode can therefore be written as

$$
\omega_{n}(k)=\sqrt{\frac{\pi^{2} n^{2}}{L^{2}}+k^{2}},
$$

with $k=\sqrt{\vec{k} \cdot \vec{k}}$.

We can rewrite the energy density from Eq. (34) in the volume contained between the plates using Eq. (36) as

$$
\begin{aligned}
E_{\text {in }} & =\frac{\mathcal{A}}{4 \pi} \sum_{n=-\infty}^{\infty} \int_{0}^{\infty} k \mathrm{~d} k e^{-\Theta \omega_{n}^{2}(k)} \omega_{n}(k) \\
& =\frac{\mathcal{A}}{2 \pi} \sum_{n=0}^{\infty} \int_{0}^{\infty} k \mathrm{~d} k e^{-\Theta \omega_{n}^{2}(k)} \omega_{n}(k),
\end{aligned}
$$

where $\mathcal{A}$ is the area of the plates and the prime on the summation in Eq. (37) means that the term with $n=0$ is weighted by a factor of $1 / 2$. Since the plates extend along $x$ and $y$ infinitely, the sums over the parallel wave vectors are replaced by integrals. We then change the integration variable from $k$ to $\omega$ and use (36) to write the energy density as

$$
E_{\text {in }}=\frac{\mathcal{A}}{2 \pi} \sum_{n=0}^{\infty} \int_{\frac{\pi n}{L}}^{\infty} \mathrm{d} \omega e^{-\Theta \omega^{2}} \omega^{2} .
$$

The energy density outside the plates can also be obtained by noting that the momentum along the $x$ direction takes continuous values there and in this region the summation over $n$ from the previous equation becomes an integral,

$$
E_{\text {out }}=\frac{\mathcal{A}}{2 \pi} \int_{0}^{\infty} \mathrm{d} n \int_{\frac{\pi n}{L}}^{\infty} \mathrm{d} \omega e^{-\Theta \omega^{2}} \omega^{2} .
$$

We again remark that the expressions for the energy density in the previous equations are finite and do not need to be regularized.

After performing the integration over $\omega$ in Eq. (38), we can calculate the force acting on the plates from inside as (recall that $\Theta=\ell^{2}$ )

$$
F_{\text {in }}=-\frac{\partial E_{\text {in }}}{\partial L}=-\frac{\mathcal{A} \pi^{2}}{2 L^{4}} \sum_{n=0}^{\infty} n^{3} e^{-\frac{\ell^{2}}{L^{2}} \pi^{2} n^{2}}
$$

and a similar expression is obtained for the force $F_{\text {out }}$ acting on the plates in the outer region with the summation over $n$ again replaced by an integration. The net Casimir force is then found by subtracting the force acting on one side of the plate from the force acting on the other side,

$$
F_{\text {net }}=F_{\text {in }}-F_{\text {out }}=-\frac{\mathcal{A} \pi^{2}}{2 L^{4}}\left[\sum_{n=0}^{\infty} n^{3} e^{-\frac{\ell^{2}}{L^{2}} \pi^{2} n^{2}}-\int_{0}^{\infty} \mathrm{d} n n^{3} e^{-\frac{\ell^{2}}{L^{2}} \pi^{2} n^{2}}\right] .
$$

We use the Euler-Maclaurin formula,

$$
\sum_{n=1}^{N} f(n)-\int_{0}^{N} \mathrm{~d} n f(n)=\frac{f(0)-f(N)}{2}+\sum_{k=1}^{\infty} \frac{B_{2 k}}{(2 k) !}\left[f^{(2 k-1)}(N)-f^{(2 k-1)}(0)\right]
$$



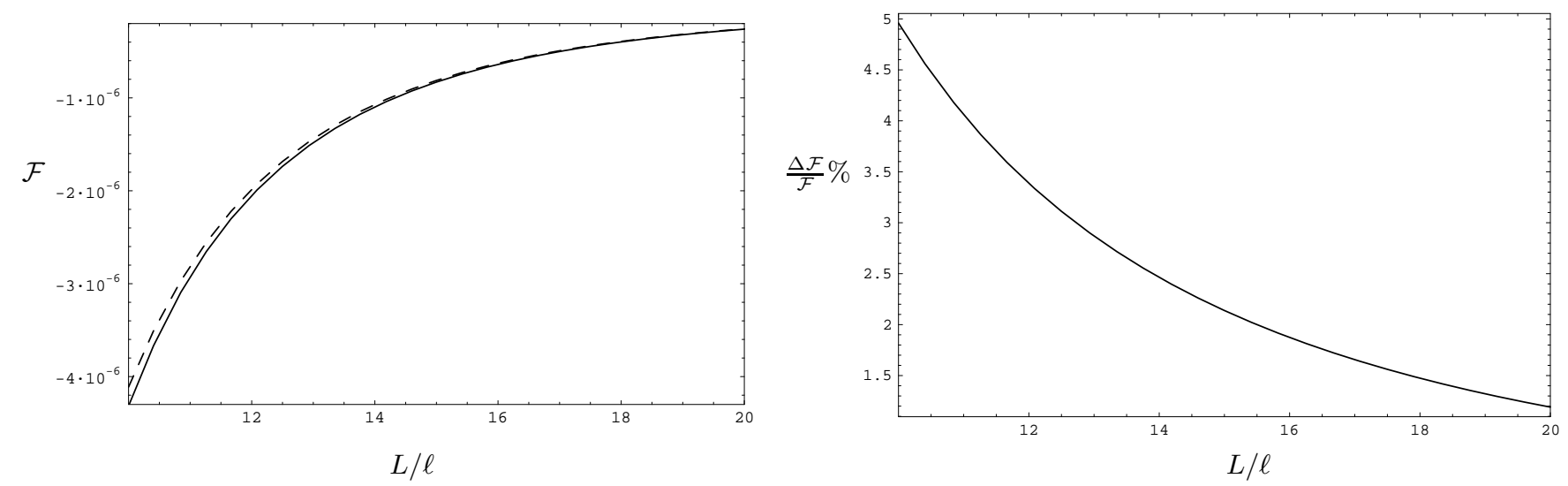

FIG. 1: Left plot: Casimir pressure in the commutative (dashed line) and non-commutative case (solid line) in units of $\Theta^{-4}$ as a function of the separation $L$ between the plates (in units of $\ell=\sqrt{\Theta}$ ). Right plot: Percentage difference between the two Casimir pressures as a function of $L / \ell$. Only volume effects are included.

where $B_{2 k}$ are Bernoulli's numbers, and the fact that in our case

$$
f(0)=\lim _{N \rightarrow \infty} f(N)=\lim _{N \rightarrow \infty} f^{(2 k-1)}(N)=0,(43
$$

to finally obtain the pressure as a power series in $\ell / L$,

$$
\begin{aligned}
\mathcal{F} & =\frac{F_{\text {net }}}{\mathcal{A}} \\
& =\mathcal{F}_{0}\left[1+\frac{17 \pi^{2}}{21}\left(\frac{\ell}{L}\right)^{2}+\frac{\pi^{4}}{4}\left(\frac{\ell}{L}\right)^{4}+\ldots\right] .
\end{aligned}
$$

The factor $\mathcal{F}_{0}$ is Casimir's original result (2), and for a comparison with the complete result including 100 orders in $\ell / L$ see Fig. 1 . For a plate separation $L \simeq 10 \ell$ the volume correction is of the order of $5 \%$ and decreases rapidly to zero for larger $L$.

We conclude this Section by noting that the leading order of the volume correction is the same $(\ell / L)^{2}$ as that of the boundary effects estimated in Section III A and, if one regards $\ell$ as the "fundamental" plate thickness, both the boundary correction (27) and the leading order volume correction (44) have the same functional dependence on $\ell / L$ as finite surface effects.

\section{COMPARISON WITH SURFACE EFFECTS}

When evaluating the Casimir force for real media, one should consider temperature corrections, corrections which appear because of the finite conductivity of the plates and because of the roughness of the plates. As we mentioned at the end of the previous Section, the latter compare very well to the type of modifications which are induced by space-time noncommutativity and will now be briefly reviewed (see, e.g., Refs. [14, 15]).

The boundaries for real plates, no matter which geometry is under discussion, show deviations from perfect figures. For example, the plates can be slightly off from being parallel to each other, or the surfaces of the plates have some imperfections. The surface roughness of any real material contributes to the magnitude of the Casimir force. In any of these cases, the amplitude of these distortions $A$ is much smaller than the separation of the two plates $L$. Even so, for plate separations of the order of $L \sim 1 \mu \mathrm{m}$, these contributions are rather large and need to be taken into consideration when comparing theoretical models with experimental data.

Let us first consider the Casimir force between two square parallel plates with sides of finite length $d$. For the derivation to be valid (we considered the case of two infinite plates) we need to have $d \gg L$. We further assume that instead of the plates being exactly parallel, they are placed with an angle $\alpha$ between them. We want $|\alpha| d \ll L$ so this angle needs to be very small (this is one of the examples of deviations from the parallel plane geometry). The correc- 
tions up to the fourth order in $|\alpha| d \ll L$ to the Casimir force in this case are given by [14]

$$
\mathcal{F}^{\alpha}=\mathcal{F}_{0}\left[1+\frac{10}{3}\left(\frac{\alpha d}{L}\right)^{2}+7\left(\frac{\alpha d}{L}\right)^{4}\right] \text {, }
$$

where $\mathcal{F}_{0}$ is again the expression in Eq. (2).

For plates covered by short scale roughness, let us describe the location of the surfaces of the two plates by

$$
\begin{aligned}
& x_{1}=A_{1} f_{1}\left(y_{1}, z_{1}\right) \\
& x_{2}=L+A_{2} f_{2}\left(y_{2}, z_{2}\right) .
\end{aligned}
$$

where $L$ is now the mean distance between the two plates. The values of the roughness amplitudes are chosen such that $\max \left|f_{i}\left(y_{i}, z_{i}\right)\right|=1$.
The mean values of the location of the plates on the $x$-axis are given by

$$
\left\langle x_{1}\right\rangle \equiv A_{1}\left\langle f_{1}\left(y_{1}, z_{1}\right)\right\rangle=0
$$

and

$$
\left\langle x_{2}\right\rangle \equiv L+A_{2}\left\langle f_{2}\left(y_{2}, z_{2}\right)\right\rangle=L \text {. }
$$

We assumed here that the amplitudes $A_{1}$ and $A_{2}$ are much smaller than the mean distance between the plates $L$, and $L$ is much smaller than both the size of the plates $d$ and their thickness $t$. At the same time in all real situations we have $L / t, L / d \ll\left|A_{i}\right| / L$ so that we are looking for a perturbation expansion in powers of $\left|A_{i}\right| / L$ to zero order in $L / t$ and $L / d$. For this case the Casimir force takes the following form [14]

$$
\begin{aligned}
\mathcal{F}^{\mathrm{R}}=\mathcal{F}_{0} & \left\{1+10\left[\left\langle f_{1}^{2}\right\rangle\left(\frac{A_{1}}{L}\right)^{2}+\left\langle f_{2}^{2}\right\rangle\left(\frac{A_{2}}{L}\right)^{2}\right]\right. \\
& \left.+35\left[\left\langle f_{1}^{4}\right\rangle\left(\frac{A_{1}}{L}\right)^{4}+6\left\langle f_{1}^{2} f_{2}^{2}\right\rangle\left(\frac{A_{1}}{L}\right)^{2}\left(\frac{A_{2}}{L}\right)^{2}+\left\langle f_{2}^{4}\right\rangle\left(\frac{A_{2}}{L}\right)^{4}\right]\right\} .
\end{aligned}
$$

In the previous sections we calculated the leading order (in $\ell / L$ ) corrections to the Casimir force due to the presence of boundaries and the volume corrections up to $(\ell / L)^{100}$ in a noncommutative scenario. We now see that, at least at leading order, the corrections due to spacetime non-commutativity have the same sign and a similar dependence on $1 / L^{2}$ as those due to the imperfections of the plates (orientation and roughness). To be more accurate, the corrections (45) and (49) due to physical imperfections of the plates depend on the square of the ratio between the amplitude of the functions which describe the deviations from perfectly flat plates and the plate separation. In the noncommutative case there are two types of corrections, one due to the "fuzziness" of the plates [estimated in Eq. (26)] and the other due to noncommutativity of space-time coordinates in the volume between the plates [see Eq. (44)]. We have shown that both of them are proportional to $\Theta / L^{2}$, where $\Theta=\ell^{2}$ is the parameter which describes non-commutativity, which can therefore be also viewed as describing an intrinsic (minimum) plate thickness.

\section{CONCLUSIONS}

In the present work we calculated noncommutative corrections to the scalar Casimir force between two parallel plates. We used a coherent states approach to non-commutativity first introduced by Smailagic and Spallucci in Refs. [8], which produces results that do not depend on the self-interaction of the scalar field.

There are two types of corrections to the attractive force between the plates. The first type of correction is what we called a "boundary" correction. Defining a boundary in the classi- 
cal sense in a non-commutative scenario is very problematic. We therefore took a heuristic approach to the problem and defined a "fuzzy" boundary which we modelled using a potential different from zero in an interval of the order of the fundamental length introduced by noncommutativity. Treating this term as a perturbation to the part of the Lagrangian which describes the interaction between the scalar field and the plates, we were able to estimate corrections which appear because of the noncommutativity of the boundaries. As a result, the scalar Casimir force is increased. We have also shown that in the limit in which the boundaries become sharp, there will still be a second contribution from non-commutativity in the volume between the plates. The non- commutativity of space-time in fact suppresses the plane waves by a Gaussian factor and thus affects the vacuum energy density. This latter contribution also has the same sign as the commutative part of the Casimir force, and we can therefore conclude that non-commutativity is in general expected to increase the (scalar and attractive) force between two parallel plates.

The treatment of non-commutative geometries with "boundaries" is a long-standing problem. The phenomenological approach we have developed here may be of use for other physical theories which involve non-commutative spaces with boundaries since it allows explicit calculations of contributions due to non-commuting aspects of space-time.
[1] H. S. Snyder, Phys. Rev. 71, 38(1947).

[2] N. Seiberg and E. Witten, JHEP 9909, 032 (1999).

[3] N. Seiberg, L. Susskind, and N. Toumbas, JHEP 0006, 044 (2000).

[4] J. W. Moffat, Phys. Lett. B491, 345 (2000).

[5] E. Witten, Surveys Diff. Geom. 7, 685 (1999).

[6] J.E. Moyal, Proc. Cambridge Phil. Soc. 45, 99 (1949).

[7] M. Wohlgenannt, arXiv:hep-th/0602105.

[8] A. Smailagic and E. Spallucci, J. Phys. A37, 1 (2004); J. Phys. A36, L517 (2003).

[9] B. Harms and O. Micu, hep-th/0610081; T.G. Rizzo, JHEP 0609, 021 (2006); A. Gruppuso, J. Phys. A 38, 2039 (2005); P. Nicolini, J. Phys. A 38, L631 (2005).

[10] R. Casadio, P. H. Cox, B. Harms and O. Micu, Phys. Rev. D 73, 044019 (2006);

[11] M. Hayakawa, Phys. Lett. B 478, 394 (2000).

[12] E. Zavattini et al. [PVLAS Collaboration], Phys. Rev. Lett. 96, 110406 (2006).

[13] H. B. G. Casimir, Proc. Kon. Nederl. Akad. Wetensch. B 51, 793 (1948);

[14] M. Bordag, U. Mohideen and V. M. Mostepanenko, Phys. Rept. 353, 1 (2001).
[15] K. A. Milton, J. Phys. A 37, R209 (2004).

[16] U. Harbach and S. Hossenfelder, Phys. Lett. B 632, 379 (2006).

[17] K. Poppenhaeger, S. Hossenfelder, S. Hofmann and M. Bleicher, Phys. Lett. B 582, 1 (2004); F. Pascoal, L.F.A. Oliveira, F.S.S. da Rosa and C. Farina, "Estimative for the size of the compactification radius of a one extra dimension universe," arXiv: hep-th/0701181.

[18] S.k. Nam, JHEP 0010, 044 (2000).

[19] From the experimental point of view, there is no compelling evidence that favors non-commutativity. We however mention that non-commutative quantum electrodynamics [11] might explain the results found by the PVLAS experiment [12].

[20] This property will be fully preserved only for volume corrections, whereas boundary corrections will depend on the coupling between the field and the plates.

[21] Note that this non-commutative term becomes of the same form as $\mathcal{L}_{\text {int }}$ in the limit $\ell \rightarrow 0$ for all values of $\lambda^{\prime}$, and one recovers the commutative theory with the effective coupling $\lambda \rightarrow \lambda+\lambda^{\prime}$. 